\title{
Visualizing changes in pretest and post-test student responses with consistency plots
}

\author{
Michael C. Wittmann ${ }^{*}$ \\ Department of Physics and Astronomy, Center for Research in STEM Education, and College of Education \\ and Human Development, University of Maine, Orono, Maine 04469, USA
}

Katrina E. Black ${ }^{\dagger}$

Department of Physics, Michigan Technical University, Houghton, Michigan 49931, USA

(Received 5 June 2013; revised manuscript received 13 March 2014; published 5 May 2014)

\begin{abstract}
Tabular presentations of student data often hide information about the switches in responses by individual students over the course of a semester. We extend unpublished work by Kanim on "escalator diagrams," which show changes in student responses from correct to incorrect (and vice versa) while representing pre- and postinstruction results on questions. We introduce the representation of "consistency plots," containing three pieces of information: each student's method of solution and correctness of solution and the shift from before to after instruction. We present data from students in an intermediate mechanics class answering (nearly) identical midterm and final examination questions. These data serve as a proof of concept of the method; we suggest other possible uses of consistency plots in physics education research, as well.
\end{abstract}

DOI: 10.1103/PhysRevSTPER.10.010114

PACS numbers: 01.40.G-

\section{INTRODUCTION}

Good representations can provide answers to difficult questions and raise questions that would not have been previously asked. In this paper, we suggest a presentation of student data on written tests that does just that: it provides rich descriptions of changes in student reasoning while raising many more questions about the nature of student learning in an intermediate (sophomore) level course on mechanics.

In physics education research (PER), we often compare student results on identical questions asked at different times. Typically, changes in responses are used to indicate learning (of a classroom or an individual student) due to some intervention designed by researchers. For example, we have preinstruction and postinstruction use of surveys like the Force Concept Inventory (FCI) [1] and Force and Motion Conceptual Evaluation (FMCE) [2]. Questions from an ungraded quiz might come back in only slightly altered form on later examinations. A question from a midterm might show up on the final examination. More generally, we often claim that questions asked before instruction as "baseline" data for studying the level of student understanding are nearly identical to later, postinstruction examination questions (see, for example, many of the citations in Ref. [3]).

\footnotetext{
*mwittmann@maine.edu

tkeblack@mtu.edu
}

Published by the American Physical Society under the terms of the Creative Commons Attribution 3.0 License. Further distribution of this work must maintain attribution to the author(s) and the published article's title, journal citation, and DOI.
We often compare students' responses on each question in order to make claims about the effectiveness of a teaching intervention. Changes in student performance (as from pre- to postinstruction tests) have typically been reported on a class average basis (see, again, many of the citations in Ref. [3]). This method of reporting has been very useful in roughly gauging the knowledge of the class when assessing the effectiveness of different kinds of curricula $[4,5]$.

However, comparing class averages can only shed light on individuals in the class when the individual response changes like the group response. When class response patterns remain static, we know next to nothing about individual student responses, though there may be great change. Bao [6] discusses the differences between reporting the class average normalized gain and the average of individual students normalized gains and suggests methods by which the differences in these two measures can yield information about the students under discussion. Separately, Bao discusses model analysis as a method of representing even static group data in which individuals give varying and internally inconsistent responses [7].

Others have attempted to address this inherent difficulty in reporting changes in student responses. The goal, as introduced by Kanim [8], was to indicate classwide shifts from correct to correct responses, without assuming that no students moved from correct to incorrect-as data regularly indicate that some do. Kanim developed the visually compelling escalator diagram (see Fig. 1) that illustrates in a compact icon both how many students responded correctly or incorrectly to a particular question at two different times and how many students changed their 
response from correct to incorrect or vice versa. The plot is read from left to right. The size of the blue and red regions on the left edge indicates how many students got the question correct or incorrect during the first asking; the size of the corresponding regions on the far right represent correct and incorrect answers during the second asking. While most students maintain their state of correctness or incorrectness in these plots, some students who were initially correct get on the red "down escalator" and give an incorrect answer later, and some who were initially wrong go up the blue escalator. The width of the diagonal escalator lines indicates how many students changed their answer [9].

Figure 1 shows three qualitative escalator diagrams. In the first, "Force/Time," more students change to correct than incorrect, leaving a net positive change in correctness. In the second and third, the total number of students answering correctly remains the same. This requires that an equal number of students change their answers from correct to incorrect and incorrect to correct between the two administrations of the test, illustrated by the equal width diagonal lines connecting the blue (correct) and red (incorrect) regions. However, in the diagram describing the "Trajectory" question, the number of students changing their answers is relatively small, whereas in the "Work" diagram, a relatively large number of students change their answers. This information would be obscured in a traditional tabular representation of the data in which it would seem that no change whatsoever had occurred. This is but a small example of a larger problem discussed by Anscombe [11], who showed how the graphing of data could highlight important differences in data sets that were not observable in the statistical values of mean, variance, and so on.

In an escalator diagram, the "red" answer can be either the most common incorrect response (leaving some "other" as white on the diagram) or it can be all incorrect answers lumped together (and the white area remains to help visualize the situation better). Van Deventer [12]

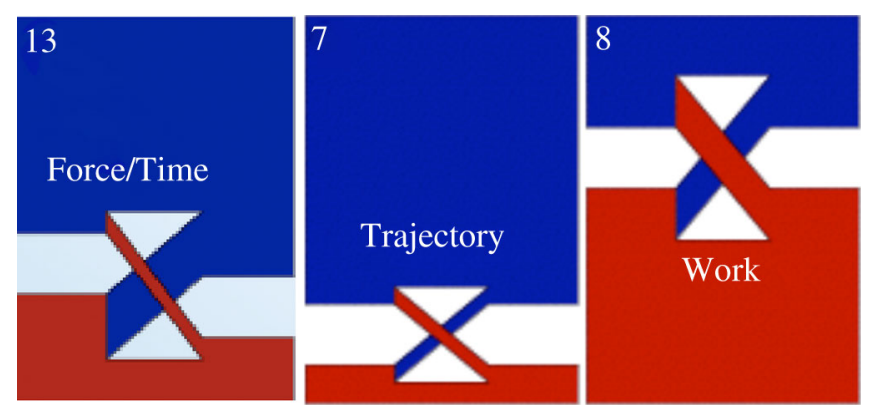

FIG. 1. Three Kanim escalator graphs, for questions about Force and Time, Trajectories, and Work. In each, percentage of students answering correctly before and after instruction is indicated, as well as change from correct to incorrect and incorrect to correct. Figures are provided by Kanim [9]. extended the escalator diagram representation to include many different responses to a question. Figure 2 shows an example from Ref. [12] on student responses to 2D vector addition questions in a physics context that came after a nearly identical question in a nonphysics "math" context.

In this paper, we take Kanim's philosophy and Van Deventer's representation and extend them to include additional information. We track individual students, like Van Deventer, and add a second dimension to the plot. This provides a far richer description of the changes in student answers and lets us discern previously unseen patterns in the changes. From this, we are able to ask questions about student learning and provide answers to questions we would not previously have asked.

Introducing a second dimension in our representation is inspired in part by work of others in PER who have done the same (see Fig. 3 for two examples). Redish et al. show scores on the Maryland Physics Expectation Survey (MPEX) [13] using two axes: a class's percentage of unfavorable responses plotted against the percentage of favorable responses. After plotting a class's scores on a given group of questions, both before and after instruction, one can draw an arrow connecting the two. The arrow can be thought of as indicating favorable movement in scores if it has a component toward the upper left-hand corner of the MPEX plots. A second example lies in model analysis plots of Bao and Redish [7]. In these, one plots a class's use of the two common models used when answering a given question. A point on the plot thus represents a class's use of each model. Again, pre- and postinstruction scores can be shown and connected with an arrow. Again, favorable motion is to the upper left on such a plot, with specific regions of the plot providing additional information described by Bao and Redish [7].

Both the MPEX plots and the model analysis plots show percentage scores as measured by surveys. The mindset of a two-dimensional plot can be extended to different data as well. In this paper, we describe the use of discrete (though overlapping) categories in which student responses can be grouped.

We are interested in problems for which there are multiple correct solutions to be studied. For example, one can use either energy reasoning or force reasoning to correctly solve certain problems in mechanics. Or, as discussed in this paper, one might use either integration limits or an undefined constant when solving an integral problem. In these and other situations, we might wish to connect student solution methods and the correctness of their solution: Are the students using method A doing better than those using method B? Do students who initially use method A incorrectly later use the same method correctly or do they change to method B? As will be shown below, these and other questions are raised (and sometimes answered) by an appropriate representation of the data. 


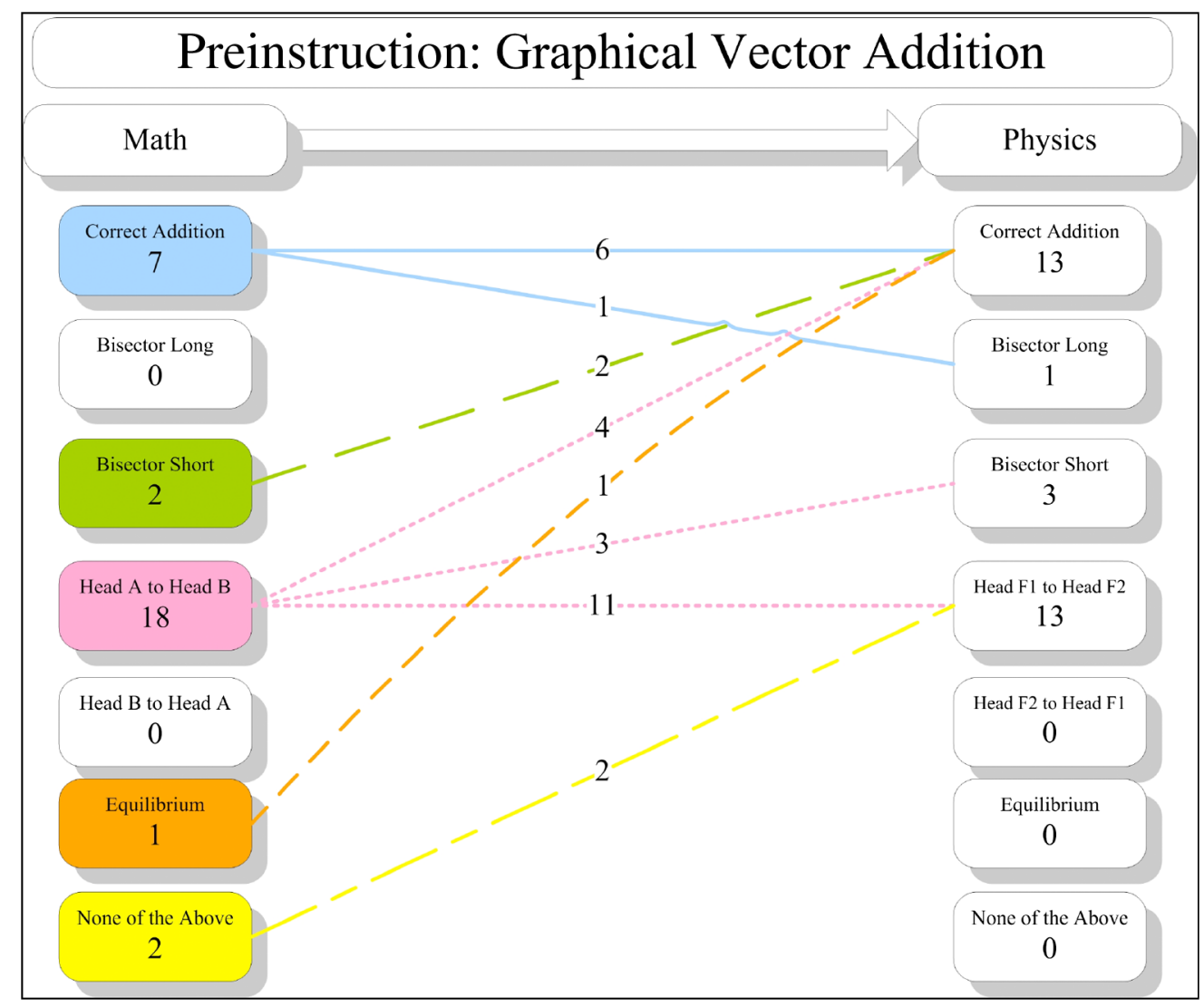

FIG. 2. Expanded escalator plot. By including all categories of partially incorrect responses (most egregious at the bottom), we gain more understanding of student responses on nearly identical questions (see Ref. [12] for more details).

The topic discussed in this paper is air resistance, of interest because it contains the relatively simple analysis of a first-order differential equation. We asked identical air resistance problems on a midterm and final exam and

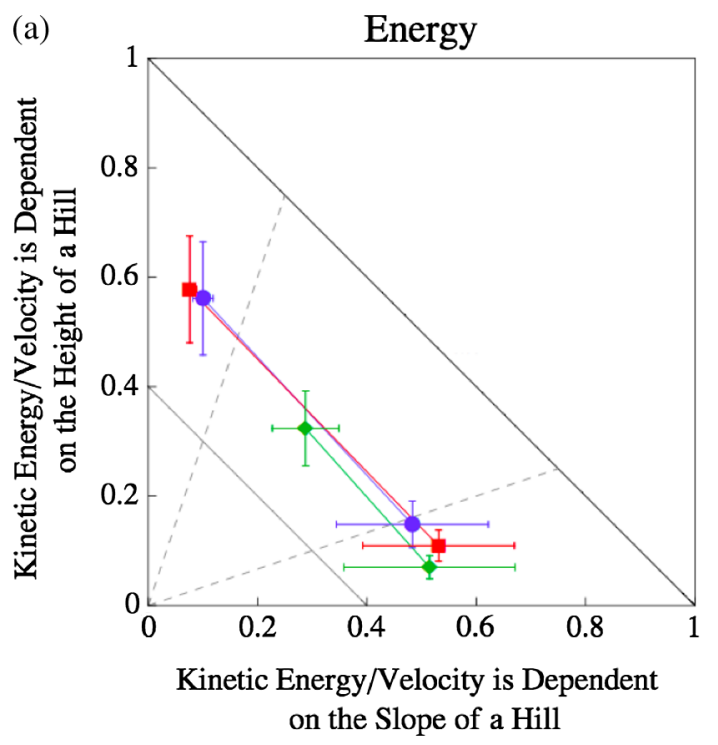

described student responses in two ways. In one description, we coded the work for the technique the student used. In another description, we coded responses based on the type of mistakes students made. To compare these two

(b)

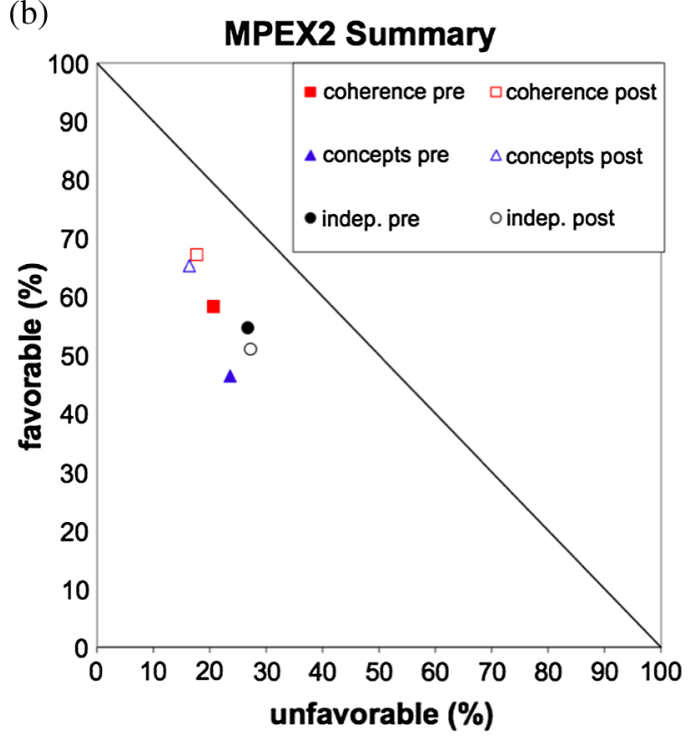

FIG. 3. Examples of 2D plots taken from research studies carried out by the authors. (a) Bao's model analysis plots show two commonly used models on a single plot (see Ref. [7] for more details). (b) MPEX plots show the class percentage of favorable and unfavorable responses to questions (see Ref. [13] for more details). 
complementary descriptions of individual student method, along with how these methods changed in time, required a more visual solution than the typical table, and was too complex to make efficient use of escalator diagrams. This led to the development of consistency plots, a kind of 2D escalator diagram. These are fully described later in the paper.

In the following section, we discuss the exam questions analyzed and our coding methodology, followed by a presentation of our results in a typical table format. We provide an example interpretation of the data based on only the tabular representation before moving on to the new representation. In Sec. III, we describe consistency plots in general and present a plot of our data. Section III A describes specific student response patterns, which are obscured by a tabular representation of the data, while Sec. III B concerns some limitations of the consistency plot representation. In Sec. IV, we suggest other measures that would benefit from being represented with consistency plots.

\section{STUDENT RESPONSES TO A PHYSICS QUESTION}

One of the first problem types in mechanics that requires the solution of a first-order differential equation is the airresistance problem for objects in a gravitational field. In such a situation, velocity-dependent forces are used to model the force exerted on a moving object. We present three years of data from an examination question that appeared both on a midterm exam early in the semester (immediately following instruction on air resistance) and again later on the final examination. In year 1, air resistance appeared in the first few weeks of the course, following a brief review of introductory mechanics. In years 2 and 3, the placement of the topic was changed. Since about half of the students in the course, every year, are taking a differential equations course in the math department concurrently (the other half have taken it previously), we wanted to allow these students to encounter the technique of separation of variables in a mathematical context before doing so in a physics context. In years 2 and 3 , air resistance was covered in the middle of the semester.

\section{A. Exam question and solution}

In year 1, the question shown in Fig. 4 appeared in a midterm exam given in week 4 of the course. In years 2 and 3 , it appeared as a group quiz in the middle of the semester. Students in years 2 and 3 worked on the problem in small groups during class time, and then solved the problem again on their own in an individual, take-home component of the quiz. We present results from these individual responses rather than the videotaped group quizzes. We look in particular at students' solutions to part $c$ of the problem [14]. The midterm problem was administered unchanged as a final exam problem in years 1 and 3; a different final exam problem (discussed below) was used in year 2 . We combine the data from all three years because we are interested in a particular aspect of the problem that was common to all the midterm and final exam administrations, namely, the use of boundary conditions to create a physically meaningful mathematical statement as a final solution.

A correct solution to the question requires several important steps. First, students must apply a correct coordinate system, with down defined as positive and perhaps the origin of the coordinate system at the launch height (note that in Fig. 6 up was defined as positive). Second, a coordinate transformation from $a=d v / d t$ to a differential based on displacement, $a=v d v / d x$, is required. In year 1, this transformation was part of a previous problem on the exam, in that students were asked which of many provided formulas correctly described acceleration (options included the given two as well as incorrect forms like $d x / d t^{2}$ ). In years 2 and 3, it was expected (and found, as shown in our video records) that students would have discussed this transformation during the group quiz preceding their individual solutions. Once the transformation had been applied and variables separated, one solves the integral, perhaps using a $u$ substitution that allows the $v$ integral to be solved with relative ease. Finally, students had to either choose the correct limits of integration (limits method) or add an integration constant and find its value ( $+C$ method). Both methods are correct, of course. An example solution of only the mathematical steps involved in using the limits method is shown in Fig. 5.

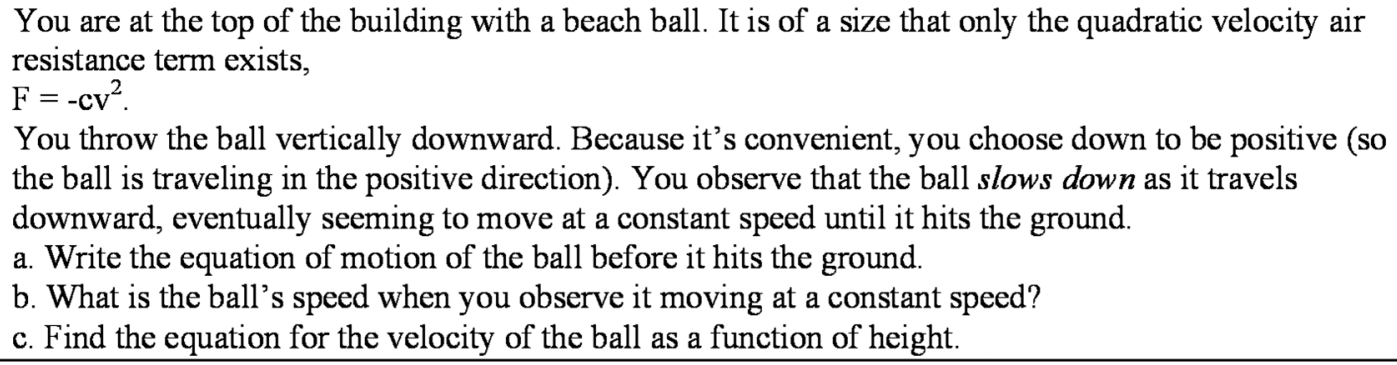

FIG. 4. Problem statement from the midterm activity in all years as well as the final examination of years 1 and 3 . Only the part of the question concerning vertical motion is shown. 


$$
\begin{aligned}
& m \frac{d v}{d t}=m g-c v^{2} \\
& m \frac{v d v}{d x}=m g-c v^{2} \\
& -m \int_{v_{0}}^{v} \frac{v d v}{c v^{2}-m g}=\int_{0}^{x} d x \\
& l e t u=c v^{2}-m g \\
& -\frac{m}{2 c} \int_{c v_{0}^{2}-m g}^{c v^{2}-m g} \frac{d u}{u}=\int_{0}^{x} d x \\
& \ln \left(\frac{c v^{2}-m g}{c v_{0}^{2}-m g}\right)=-\frac{2 c}{m} x \\
& v=\sqrt{\left(v_{0}^{2}-\frac{m g}{c}\right) e^{-\frac{2 c}{m} x}+\frac{m g}{c}}
\end{aligned}
$$

FIG. 5. Possible problem solution using the limits method. Written explanations have been omitted for brevity.

In our problems, the magnitude of the initial velocity was not given explicitly and had to be defined by the students as $v_{0}$ or something similar. Also, the question asked for students to give the velocity as a function of height. Although this might have impaired some students' reasoning, evidence from video recordings of the group quizzes (not discussed in this paper) suggests that this atypical definition was not generally problematic for the students, who seemed to use height and distance interchangeably.

In year 2, the final examination question was modified (see Fig. 6). Students were asked to find the velocity as a function of time rather than position, and the air resistance force was $v$ and not $v^{2}$ dependent. More consequentially, students were told to consider up as the positive direction but to consider both upward and downward motion. This slight perturbation led to a large effect in the data, in that sign errors with changes of velocity were far more common. The differences in the questions require at times that we exclude or record separately the results from this year. We will indicate each time this is the case. The dependence of student reasoning on simple changes to coordinate systems is an interesting point, and consistent with other results from our studies on student use of coordinates and coordinate systems, but outside of the scope of this paper [15,17-19].

Because we are discussing changes in individual student responses, we will consider only those students (in all three years) for whom we have matched data. We collect data from all three years, in spite of the questions being asked in different formats in the given years, because we are interested in the general question of how students use boundary conditions when solving integration problems. Though we suspect that question format may affect this issue, we emphasize that the representation we introduce is the primary topic of this paper, rather than the specifics of question administration.

\section{B. Tabular presentation of student answers}

We have gathered data from three years of instruction. Solution method categories arose from the data, being those methods most commonly used. Most students used separation of variables to solve the differential equation. These students were placed into one of the three groups: the limits method modeled in our physics class, the $+C$ method familiar to our students from math classes, or a group containing those students who applied both methods to the problem. Those students who did not use separation of variables (including two students who applied the more complex technique of variation of parameters with an integrating factor) as well as students who used separation of variables but did not use limits or an integration constant (essentially, only finding the antiderivatives) were categorized as "other" solutions. As might be expected, most students who only found the antiderivatives or did not use separation of variables were unable to progress far into the problem.

We also grouped solutions according to correctness.

$(\bullet)$ "Correct" solutions were carried out mathematically correctly and related the mathematics to the physical meaning by successfully including the initial conditions in the final statement of the solution.

\footnotetext{
You stand at the edge of a cliff on the mountain and throw two balls at the same time: Ball A vertically downward at greater than terminal velocity ( speed $v_{0}>v_{t}$ ) and Ball B vertically upward at greater than terminal velocity $\left(\right.$ speed $v_{0}>v_{t}$ ). Both balls experience an air resistance force proportional to velocity, $v$. For convenience sake, let $+y$ be in the upward direction.

A. Write the differential equation that describes the motion of the ball thrown upward.

B. How, if at all, will your differential equation change for the ball thrown downward? Explain. If there is no change, state so explicitly.

C. Write the equation for $v(t)$ for Ball A (the one thrown downward). Show all work
}

FIG. 6. Year 2 final exam problem. This problem used a different velocity-dependent force and different question format from the year 1 and 3 problem but still required students to solve an integral while appropriately using initial conditions for a fully correct response. 
(•) Problems with a "math error" could include simple algebraic mistakes or might progress to difficulties using a $u$ substitution. These were, in our mind, errors of a procedural nature, typical to solving mathematics problems, rather than errors of a conceptual nature related to the physics. (We acknowledge that mathematics education researchers might find conceptual meaning in many of these errors.) Students who failed to consider the domain of the natural log function were also placed in this group, even though the reasoning required to determine the sign of the argument of the ln function was physical. For these students, the error arose in that the argument of the ln function can be negative, and therefore the function is undefined in an intermediate step of the problem. Few students recognized this, indicating that they were in an algorithmic, and not mathematical, mindset while working on this part of the problem.

$(\bullet)$ Those students with a "boundary condition error" might use unphysical limits, set $v_{0}=0$ later in the problem, or leave the integration constant undefined, simply as $+C$.

(•) Finally, students in the "other" category either did not come to a final answer or made a serious physical error while setting up the problem (such as writing terms in the equation in a way that contradicted the given coordinate system [15]).

The categories chosen and used in this papers serve as an example for how one might use consistency plots by grouping student responses in meaningful ways and plotting changes over time. Other categories could have been used (as discussed below). The categories are consistent with our goal to observe how students used boundary conditions to give physical meaning to mathematical statements. Therefore, we defined the boundary condition error category very narrowly and defined the math error and other categories quite broadly. For our specific purposes, math errors of any type were less serious than boundary condition errors because the latter depend on the students' physical reasoning abilities while the former may simply be evidence of a careless math error. Different research goals (say, on the use of coordinate systems and their role in translating physical systems into mathematical statements [15,17-19]) would have led to different categories. Such categories would have led to different consistency plots, which we do not show in this paper in the interest of space.

Notably, the categories described above are not mutually exclusive. A student might make a math error and also not apply the boundary conditions of a problem. We have counted these errors in both locations on the table (for example, in year 1 midterm data, more than 15 responses are indicated in the table). Again, other research interests might have led to other categorizations, which does not take away from our overall argument.

Major results of these groupings are shown in Tables I and II.

\section{Apparent conclusions: A short interlude}

We step back from our presentation of the problem and the data to consider an analysis of the data, using only the tables provided thus far. For these purposes, we combine all years' data into a single table, as might be done to improve statistics when analyzing a course with such low yearly populations and the same general instructional techniques. We observe in Table I that the solution methods students use remain fairly static, with only a change of one or two students per category. Though we emphasize using the limits method in the course, many students seem to stick to the more familiar $+C$ method that they originally learned in their mathematics courses. These trends hold across all three years of instruction.

TABLE I. Student solution techniques. Students are grouped by their approaches to solving the required differential equations.

\begin{tabular}{|c|c|c|c|c|c|c|c|c|}
\hline \multirow[b]{2}{*}{ Category } & \multicolumn{2}{|c|}{ All years $(N=39)$} & \multicolumn{2}{|c|}{ Year $1(N=15)$} & \multicolumn{2}{|c|}{ Year $2(N=10)$} & \multicolumn{2}{|c|}{ Year $3(N=14)$} \\
\hline & Midterm & Final & Midterm & Final & Midterm & Final & Midterm & Final \\
\hline Used only limits & 24 & 22 & 7 & 8 & 7 & 5 & 10 & 9 \\
\hline Used only $+C$ & 5 & 3 & 2 & 1 & 0 & 0 & 3 & 2 \\
\hline Used limits and $+C$ & 3 & 2 & 3 & 1 & 0 & 1 & 0 & 0 \\
\hline Other & 10 & 12 & 6 & 5 & 3 & 4 & 1 & 3 \\
\hline
\end{tabular}

TABLE II. Student responses on midterm and final exams. Students can be listed in multiple categories.

\begin{tabular}{|c|c|c|c|c|c|c|c|c|}
\hline \multirow[b]{2}{*}{ Category } & \multicolumn{2}{|c|}{ All years $(N=39)$} & \multicolumn{2}{|c|}{ Year $1(N=15)$} & \multicolumn{2}{|c|}{ Year $2(N=10)$} & \multicolumn{2}{|c|}{ Year $3(N=14)$} \\
\hline & Midterm & Final & Midterm & Final & Midterm & Final & Midterm & Final \\
\hline Correct & 6 & 7 & 1 & 2 & 0 & 0 & 5 & 5 \\
\hline Math error & 28 & 18 & 11 & 10 & 10 & 4 & 7 & 4 \\
\hline Boundary condition error & 15 & 16 & 7 & 6 & 3 & 9 & 5 & 1 \\
\hline Other & 4 & 18 & 4 & 6 & 0 & 6 & 0 & 6 \\
\hline
\end{tabular}


Table II tells a slightly different story, but one that is unfortunately familiar to many instructors: few students moved into the correct category from the midterm to the final exam, but, in contrast, many students' solutions change so much that they must be categorized as "other." This increase in the "other" category happens most strikingly in years 2 and 3. While the number of math errors decreases, the number of boundary conditions errors remains fairly constant (though only in the aggregate, since there are large differences between years 2 and 3).

Based on these data, as represented in these tables, an observation might be as follows:

Instruction did not significantly change the method students use to solve air-resistance problems. While on both midterm and final exams about $15 \%$ of students are correct, more than twice that many make boundary condition errors, indicating a disconnect between the mathematical method of separation of variables and the physical reality it represents. Regrettably, students with a deeply flawed (categorized as "other") solution increase from $10 \%$ to $45 \%$.

From these observations, we might pursue several different conclusions. Perhaps the group work preceding the written tests in years 2 and 3 affected midterm performance and lowered the "other" responses on the midterm. Perhaps on the final exam in years 2 and 3 students simply had too much to study for and were more careless in setting up problems. Perhaps problems with differential equations were not a large part of the course content in the last third of the semester, so students were unprepared for such questions on the final. Other conclusions are available, as well.

Interesting as these hypothetical observations and conclusions might be, more fundamental concerns should be addressed first. The tallies shown in Tables I and II are incomplete because they cannot tell how the solutions of individual students change over the course of the semester. Are those the same five stalwart students getting the right answer on the midterm and final exams in year 3, or did those five change their answers while five new students answered correctly? In year 1 , fewer students used the "limits and $+C$ " method on the final exam, but what happened to those students who changed their answer?

A multitude of escalator plots might be created to address these concerns and questions. Answers have serious consequences when considering how to improve teaching. If students who start correct stay correct, we can spend our time focusing on those who need the most help. If correct students change their answers, then we have to worry about how to help them stay where we wish them to stay. Answers also have serious consequences when considering our research. If students are not answering consistently over time, we should question conclusions that are too general, if they are drawn from a single test or observation of student work. Some of these concerns are addressed and made clearer when the data are presented in a consistency plot.

\section{CONSISTENCY PLOTS REPRESENT MULTIPLE DIMENSIONS OVER TIME}

To show how student responses change over time, we introduce a graphic we call a consistency plot. We draw a 2D grid representing two separate ways of analyzing student responses. Each student whose answers are different from the midterm to final exam is shown by an arrow formed of a circle, a line, and a triangle. A circle represents an initial (midterm) response; a triangle represents a final response. This circle-line-triangle grouping is referred to as a response pair and describes the two solution states of a single student at two different times. A square represents a response pair in which the initial and final responses are identical. Both the size of the element and the number placed within it reveal how many students had that particular response pair. The plot axes can represent any two lines of analysis of the data. The term "consistency plot" is used to focus the attention on how consistently students answered at two different times.

In our case (see Fig. 7), we use the previously discussed categories of solution method and correctness along each axis. We present only data from years 1 and 3. Year 2 data were omitted due to the difference in initial and final question statements and will be discussed in Sec. III B.

The plot shows the considerable variety in how student responses changed with time. Most response pairs describe lone individuals. Only five groups of response pairs are made up of more than one student:

(•) Three students use limits and are correct on both midterm and final, as represented by a box inside (limits, correct)

(•) Three use limits and have a math error on both the midterm and final, as represented by a box inside (limits, correct)

$(\bullet)$ Two pairs of students leave the (limits, math error) and one pair leaves (limits, correct) on the grid.

In total of the 29 student response pairs in years 1 and 3, 22 (about three quarters) are unique to a single student. This variability in student responses indicates that there is much more richness and change than indicated in Tables I and II.

\section{A. Elements of consistency plots}

It is in the nature of consistency plots to be at least somewhat messy; the richness of information that they present can look chaotic at first glance. However, patterns do emerge from the plots. Figure 8 shows an idealized consistency plot that contains four elements we have found in our data. We discuss these four elements before returning to the data shown in Fig. 7. 


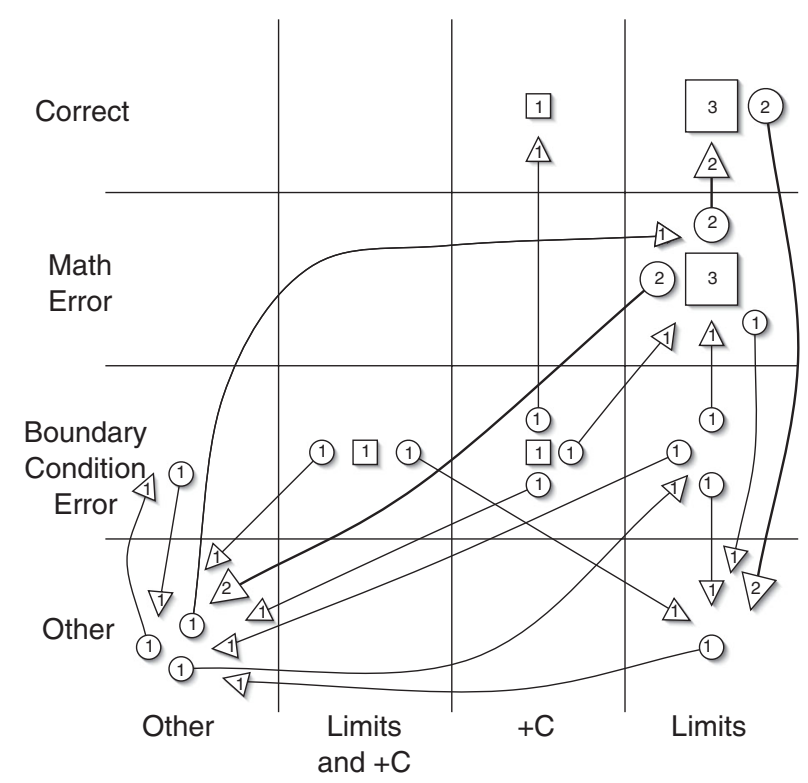

FIG. 7. Consistency plot showing changes in student midterm performance and final exam responses to identical questions in both years 1 and 3 . Note that year 1 students had a midterm exam and year 3 students answered questions individually after engaging in a group quiz. Final exam questions were identical.

$(\bullet)$ Attractor: The upper right-hand corner of the plot (limits, correct) is attractive; that is, many more response pairs end there than begin there.

(•) Starburst: The region (limits and $+C$, boundary condition error) is an example of a starburst, where student responses leave a space in many directions.

$(\bullet)$ Circulation: To the far left we see circulation, where two regions are connected by opposite response pairs.

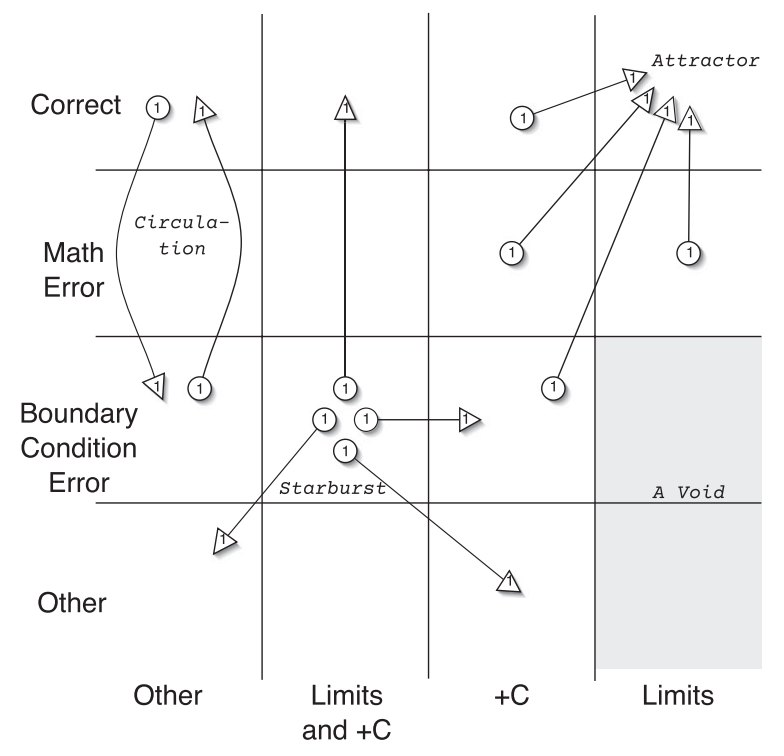

FIG. 8. Sample consistency plot (i.e., not actual data) showing examples of attractors, starbursts, circulation, and void.
(•) Void: Finally, in the bottom right, there is a void, a region with no responses.

As is apparent from our plot of actual data in Fig. 7, real life is not so accommodating as to provide easily observable attractors, starbursts, circulators, or voids. But we can use the terminology to discuss what is otherwise a very complicated representation of data.

Below, we present excerpted examples of each of these elements, taken from our year 1 and 3 consistency plot. For clarity, we will show each element on a separate plot, along with comments regarding the implications of each element for this particular question. Before reading on, however, the reader may find it interesting to return to Fig. 7 to find an example of each grouping.

Figure 9 shows three examples of circulation, that is, sets of opposite response pairs. A tablelike approach to the data would state that, for example, one student on the midterm and final exams used an "other" method while making a boundary condition error. In fact, our consistency plot shows that this data point is made up of two students, each of whom at one time or another was also in the (other, other) category. Similarly, while a table would show a net flow (of one for the data presented on this subplot) into the "other, other" category, it would not show that three students improved their responses. Circulation is the 2D version of an effect also found in Kanim's escalator diagram, namely, that nontrivial numbers of students may switch from one answer to another, and vice versa, over the course of instruction. Examples of circulation are of interest because they are undetectable in a traditional reporting of data, but suggest a fluidity in learning that

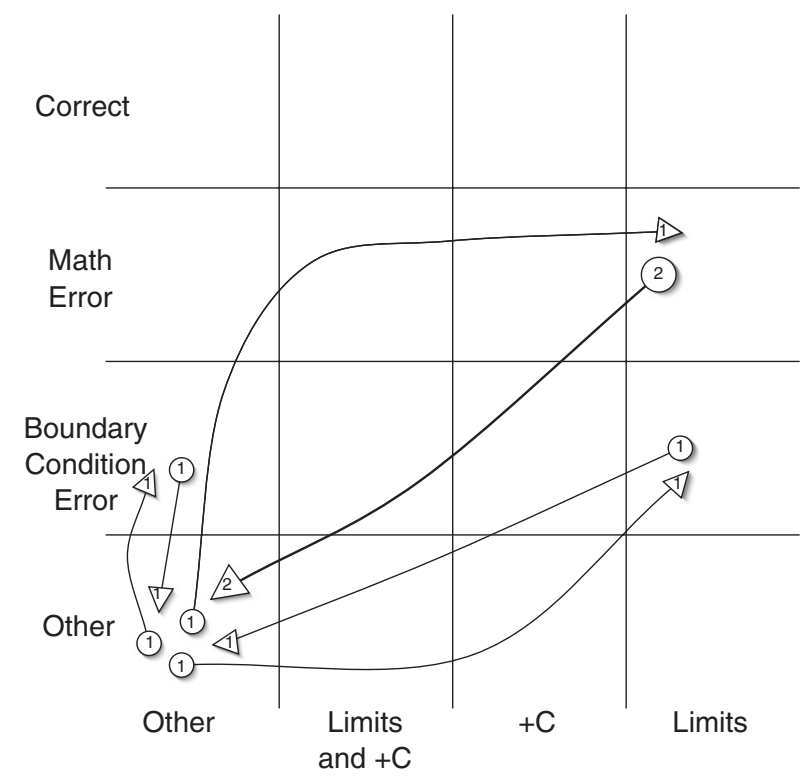

FIG. 9. Circulation pattern representing a subset of data from Fig. 7. Students flow out of and into matched regions on the consistency plot. 
offers opportunity for further study (for example, with response curves [20]).

In reality, there was much more flow into the (other, other) region than out of this region (see Fig. 10). We call such a region an attractor. In our idealized plot, no responses left the region; in this real-world example, three leave, giving a net flow of four students. Seven students found themselves in this category on the final exam. Yet all but one of the midterm solutions used a separation of variables technique, and two contained only math errors. In other words, most of the students who performed badly on the final exam gave little forecast of this eventuality on the midterm exam.

Although we would much rather see the (limits, correct) region be the attractor (as it was on our idealized plot, Fig. 8), this attractor makes visual the unfortunate case of students who succeeded on the midterm and then performed far worse on a final examination. Such data require further investigation and were not readily apparent from either Table I or II.

Several small starbursts appear in Fig. 7, our year 1 and 3 consistency plot. For visual clarity, we indicate only two in Fig. 11, with origins in $(+C$, boundary condition error) and (limits and $+C$, boundary condition error). In each case, two important aspects are apparent: students do not enter the region, and the students that leave go in many directions rather than to another specific region. [In a third starburst, from region (limits, boundary condition error), a student does enter that region, as well.]

Had the students all gone in the same direction, we might infer that the indicated region was acting as a "stepping stone" along the learning path toward a more refined solution. Instead, we see that these students made a

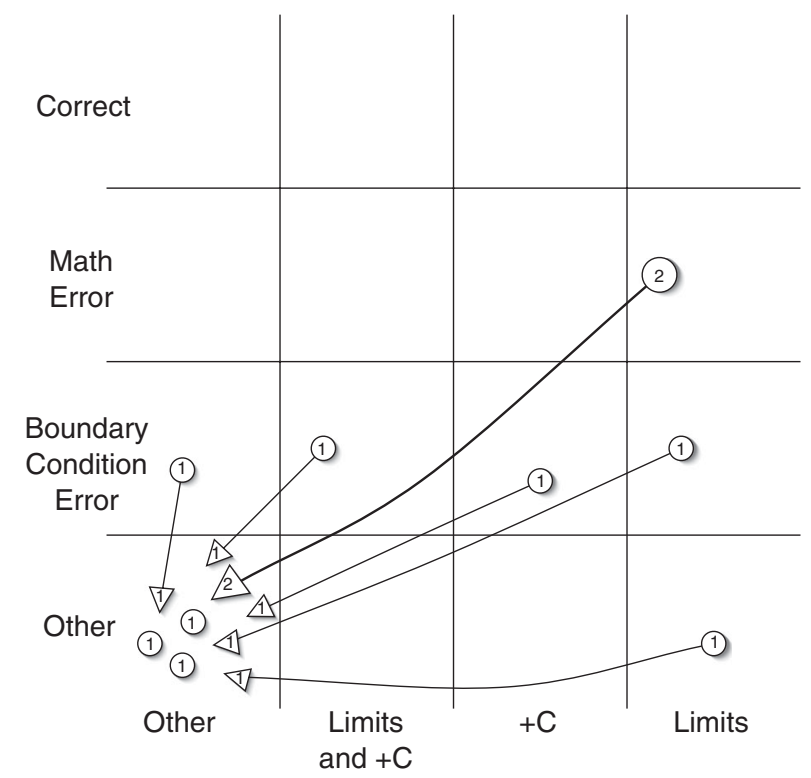

FIG. 10. Attractor pattern representing a subset of data from Fig. 7. Students move toward one region on the consistency plot.

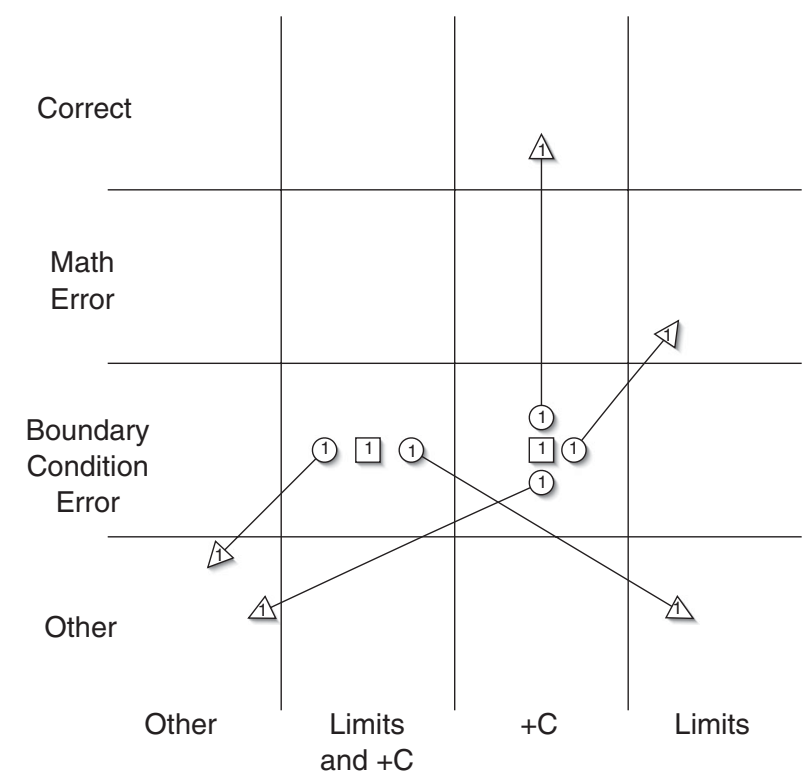

FIG. 11. Starburst pattern representing a subset of data from Fig. 7. Nearly all students leave a given region on the consistency plot. Note that this plot shows examples only, and that not all starbursts in Fig. 7 are indicated.

consistent kind of error on the midterm exam and solved the problem very differently on the final exam. Because students went in many directions, we suggest that students were using unrefined thinking, that is, were using every mathematical tool available to them, indiscriminantly.

We can see the difference between the "every tool" and the "stepping-stone" interpretation by looking more carefully at the starburst starting in the (limits and $+C$, boundary condition error) region. Three students use both limits and an integration constant in their solutions on the midterm exam. When we first ran across this method on the midterm, we interpreted it as something students might do when trying to apply their usual method of using integration constants, the mainstay of math classes, while trying to satisfy their physics instructor, who consistently modeled the use of integration limits in class. Although mathematically sketchy (since, of course, limits and an integration constant serve the same mathematical role), we thought that it was simply a sign of students becoming used to the limits method and including it in their solutions without yet recognizing that the two methods are mathematically equivalent. We suspected that if this were the case, these students would later solely use the limits method as they became more comfortable with it. Had our assumptions been correct, these three students would have all moved toward the (limits, correct) region. They did not. Instead, the starburst indicates that these students were simply using all the techniques available to them on both the midterm and the final exams. Again, the consistency plot provides information and suggests interpretations that were not available using a tabular presentation of the data. 


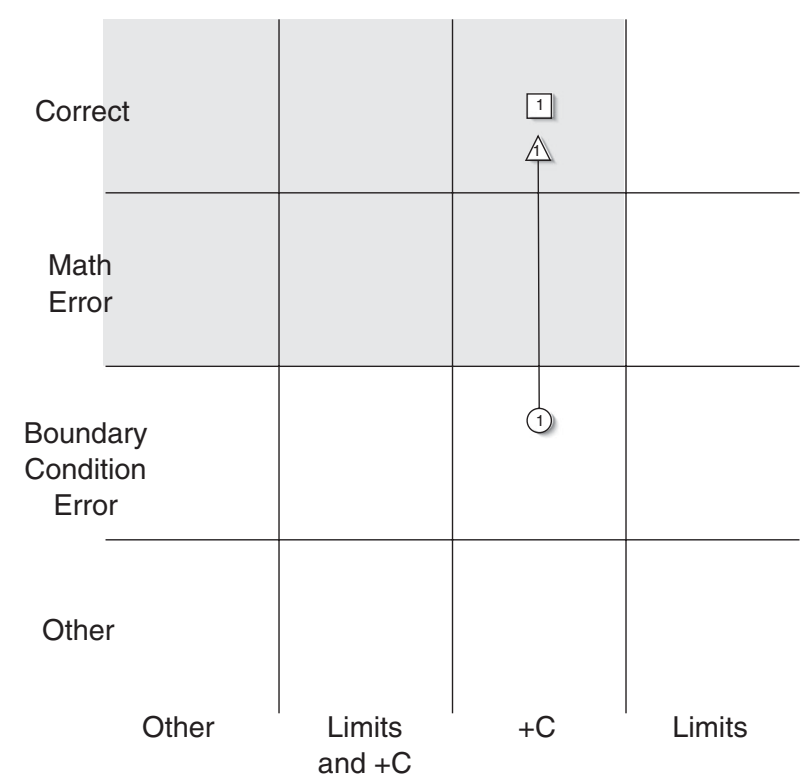

FIG. 12. Void pattern. Nearly no answers are found in an extended region of the consistency plot.

Finally, in Fig. 12 we consider the highlighted void region where few student responses are located. There are 21 solutions in the region below the void, representing students who used a method other than limits and either did not successfully incorporate the boundary condition into the problem or were unable to appropriately represent the physical situation mathematically. Of 29 students over two exams in two years, only two (a total of three times) use an integration constant and also find its value using the boundary condition. No students were correct when using a method other than separation of variables, though other appropriate techniques exist [21]. Of course, zeros show up in data tables, but the visual nature of the consistency plot makes the regions that contain few students as compelling as the regions that contain many students, and leads us to consider what students are not doing along with what they are doing. Since we are looking at how students bring physical meaning to the mathematics, the fact that only two of 29 students use a method other than the limits method to correctly and completely solve the problem is noteworthy for instructors helping students learn the physics (and mathematics) better.

\section{B. Possible weaknesses of a new representation}

As we have shown, our representation can allow a deeper analysis of data than a simple table. We see how students change with time, which solution methods are used less or more often, and which combinations of solution and correctness are rare or nonexistent. These are all advances over a tabular representation of classroom data.

However, consistency plots are not without weaknesses. As an example, we present a consistency plot of student responses during year 2. In this year, the problem was changed on the final exam so that the positive direction was up (see Fig. 6). In Fig. 4, positive was down. Our goal in reversing the direction was to give a subtly different problem from the midterm, so that memorized responses could not be used as readily. The change in coordinate system leads to a change in the definition of the initial velocity of the downwardly thrown ball, among other things. Since, by convention, physical constants are positive, students needed to define the initial velocity of the ball as $-v_{0}$. The change also caused serious problems for students not setting up the problem correctly; their translation of the physical system into coordinates often included the wrong signs. Such errors were considered "other" errors (in keeping with our previous definitions). Because the seriousness of the error often prevented them from making it further into the problem, students making "other" errors would not be counted as making boundary condition or math errors. As a result, the goal of keeping categories consistent across different questions leads to interpretation of the data which might not do justice to student work; these problems are not unique to consistency plots but are visually more apparent due to the nature of a visual representation.

An initial perusal of Fig. 13 suggests that only three students ended in the boundary condition error row of the diagram. However, the hierarchical nature of our vertical axis obscures the fact presented in Table II that nine students actually made the $-v_{0}$ error. Because six students made errors in the setup and execution of the problem that were severe enough to classify as "other," we lose information about the prevalence of boundary condition errors in the year 2 consistency plot.

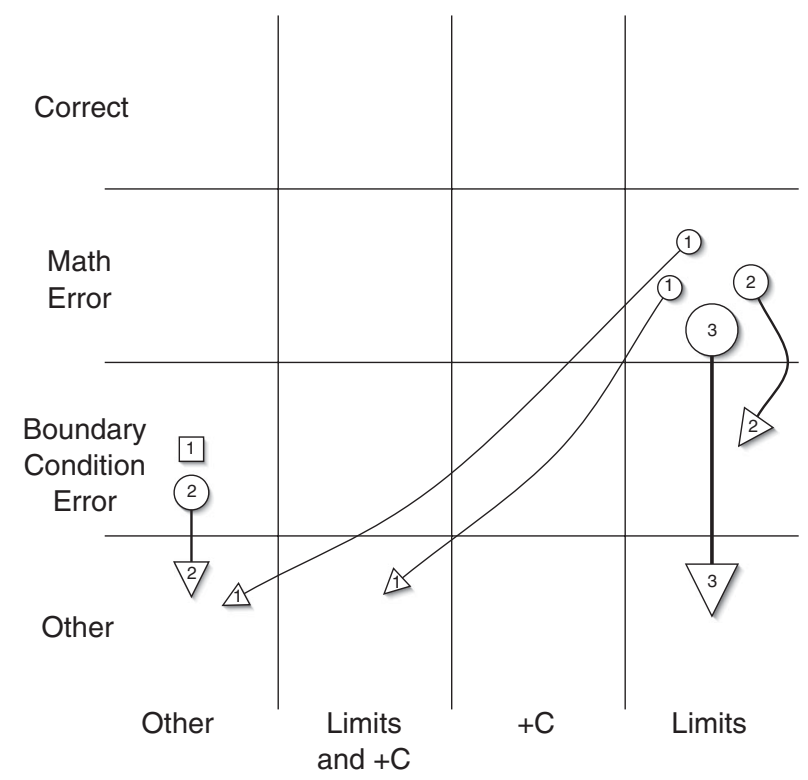

FIG. 13. Consistency plot from year 2. Final examination question had a differen coordinate system, leading to many "other" responses that hide information from further analysis. 
In part, this situation can be alleviated by careful attention to coding and the refinement of coding schemes. (For example, choosing $v_{0}$ rather than $-v_{0}$ might be called a boundary condition error because it is an incorrect mathematization of the boundary condition.) However, we cannot always predict in advance what the data might say, and there is always the potential that a hierarchical coding scheme will conceal interesting patterns, so we do not recommend the abandonment of the data table. One might allow for fuzzy categories, but having one student in two regions seems impossible to interpret visually. One might create more detailed exclusive categories, but developing too many categories to eliminate a hierarchical grouping and describe student responses more fully may create a plot too chaotic to read. We already have very few students doing the same thing from midterm to final exam - to use further detail to separate the responses would make the data (including observation of attractors and starbusts) nearly meaningless for small classes. The balance between inclusiveness of categories and visual accessibility will be specific to each data set, and so must be determined on an individual basis. We have chosen to keep the year 2 consistency plot on the same scale as the year 1 and 3 plot, for example.

\section{DISCUSSION}

We have introduced consistency plots, a twodimensional method of presenting student responses and how they change over time. As a visual presentation of data, they allow the recognition of patterns of student response that may not be available or easily discernable when the data are presented in tabular form. As an example, we have used the plots to discuss how students' solution methods and correctness for solving first-order separable differential equations change from a midterm to a final exam. We believe, though, that the method is far more general and could be used to analyze much larger sets of data as well as very different kinds of categories.

We show several examples of interesting response patterns within the overall plot: attractors, starbursts, circulation, and voids. Each is revealing about student performance in the class, yet most are hard to represent in simple tabular form. Attractors can show where students are being led over the course of instruction while also indicating what these students did previously. Starbursts help us distinguish between solutions that indicate students developing toward new ideas (i.e., stepping stones, where all students move to the same answer on the final) and solutions that indicate students' confusion about the problem (where student methods go in many directions). Circulation calls into question how one uses classwide tables of data to imply improvement, or lack thereof, in student performance. Voids indicate a "failure" to use some method in a specific way. These elements of classroom response patterns suggest that consistency plots can be a very useful tool for investigating the change in student responses in a course and are worthy of further exploration and implementation.

We also note difficulties with the use of consistency plots. When the hierarchical categorization of data is employed, some patterns of student response may be obscured. The inability to use fuzzy categorization, i.e., requiring students to be in only one region or category, is another problem. Student responses are often richer than the simple placement on a single point on a plot can represent. Thus, consistency plots are limited by the researchers' assumptions and interpretations of the data. Our interests in this paper were in students giving physical meaning to mathematical solutions, and this affected our choices about categories and assignment of students to given categories. These specific choices should not detract from a discussion of the general utility of consistency plots. One could, for example, create more clearer categories by using multiple choice questions to guide the groupings along one or both of the axes.

Consistency plots could serve as a rich tool for further investigation of pre- and post-testing in PER. Given data from the FMCE [2], one could plot different categories of student responses (say, a scale related to Newton's second law on one axis and a scale related to acceleration questions on another, as suggested by Springuel et al. [22]) and track students' responses over the course of the semester. Such a representation would provide information about students' development, based on their incoming knowledge. A similar plot of FMCE versus MPEX [13] might allow researchers to make connections between gains on content surveys and student attitudes toward physics.

We believe consistency plots can be useful for displaying data in a wide variety of situations. Whenever individual student responses to pre- and post-test questions are being considered, it is likely that consistency plots will be a useful addition to standard tables for interpreting patterns of student response.

\section{ACKNOWLEDGEMENTS}

Work supported in part by NSF Grants No. REC0633951 and No. DUE-0962805. 
[1] D. Hestenes, M. Wells, and G. Swackhamer, Force Concept Inventory, Phys. Teach. 30, 141 (1992).

[2] R. K. Thornton and D. R. Sokoloff, Assessing student learning of Newton's laws: The Force and Motion Conceptual Evaluation and the Evaluation of Active Learning Laboratory and Lecture Curricula, Am. J. Phys. 66, 338 (1998).

[3] L. C. McDermott and E. F. Redish, Resource Letter PER-1: Physics Education Research, Am. J. Phys. 67, 755 (1999).

[4] R. R. Hake, Interactive-engagement versus traditional methods: A six-thousand-student survey of mechanics test data for introductory physics courses, Am. J. Phys. 66, 64 (1998).

[5] E. F. Redish, Millikan Award Lecture (1998): Building a science of teaching physics, Am. J. Phys. 67, 562 (1999).

[6] L. Bao, Theoretical comparison of average normalized gain calculations, Am. J. Phys. 74, 917 (2006).

[7] L. Bao and E. F. Redish, Model analysis: Representing and assessing the dynamics of student learning, Phys. Rev. ST Phys. Educ. Res. 2, 010103 (2006).

[8] Steven Kanim (private communication).

[9] In many ways, these escalator diagrams are consistent with and simple versions of Sankey diagrams of, for example, energy flow in our power grid (previously discussed in a PER context by Scherr et al. [10]). In such diagrams, the width of the line is proportional to the flow, just as in the escalator diagrams.

[10] R. E. Scherr, H. G. Close, S. B. McKagan, and S. Vokos, Representing energy. I. Representing a substance ontology for energy, Phys. Rev. ST Phys. Educ. Res. 8, 020114 (2012).

[11] F. J. Anscombe, Graphs in statistical analysis, Am. Stat. 27, 17 (1973).
[12] J. Van Deventer, Masters thesis, University of Maine, 2008.

[13] E. F. Redish, J. M. Saul, and R. N. Steinberg, Student expectations in introductory physics, Am. J. Phys. 66, 212 (1998).

[14] We note that issues related to the videotaped group quiz are discussed elsewhere $[15,16]$ and deal with issues related to coordinate systems and the development of the skills for separating variables, not discussed in detail in this paper.

[15] K. McCann and M. C. Wittmann, The role of sign in students' modeling of scalar equations, Phys. Teach. 48, 246 (2010).

[16] K. E. Black, Ph.D. thesis, University of Maine, 2010.

[17] E. C. Sayre, M. C. Wittmann, and J. E. Donovan, Resource plasticity: Detailing a common chain of reasoning with damped harmonic motion, AIP Conf. Proc. 883, 85 (2007).

[18] E. C. Sayre and M. C. Wittmann, Intermediate mechanics students' coordinate system choice, in Proceedings of the Conference on Research in Undergraduate Mathematics Education, edited by M. Oehrtman (SIGMAA, San Diego, CA, 2007).

[19] E. C. Sayre and M. C. Wittmann, Plasticity of intermediate mechanics students' coordinate system choice, Phys. Rev. ST Phys. Educ. Res. 4, 020105 (2008).

[20] E. C. Sayre and A. F. Heckler, Peaks and decays of student knowledge in an introductory E\&M course, Phys. Rev. ST Phys. Educ. Res. 5, 013101 (2009).

[21] One might recall the form of the solution of the differential equation or use the more complex technique of variation of parameters.

[22] R. P. Springuel, J. R. Thompson, and M. C. Wittmann, Using cluster analysis to group student responses on the Force and Motion Conceptual Evaluation (to be published). 